\title{
Frizzled class 7 receptor is differentially expressed in high-grade serous ovarian cancer
}

and based on patient survival.

Shahan Mamoor, MS 1

1shahanmamoor@gmail.com

East Islip, NY USA

High-grade serous ovarian cancer (HGSC) is the most common type of the most lethal gynecologic malignancy ${ }^{1}$. To identify genes whose expression was associated with survival outcomes in HGSC, we used published data from patients enrolled in the ICON7 trial to compare the global gene expression profiles of primary HGSC tumors from women with the best and worst progression-free survival (PFS) ${ }^{2}$. We found that the Frizzled class 7 (Fzd7) receptor was among the genes most differentially expressed in HGSC tumors when comparing tumor transcriptomes based on superior or inferior PFS. In two independent datasets, Fzd7 was among the genes most differentially expressed in HGSC tumors when comparing primary tumor to the normal ovary ${ }^{3,4}$. Wnt pathway signaling through Fzd7 may be relevant to the biology of highgrade serous ovarian cancers.

Keywords: ovarian cancer, high-grade serous ovarian cancer, HGSC, targeted therapeutics in ovarian cancer, systems biology of ovarian cancer, Fzd7.

PAGE 1 OF 14 
High-grade serous ovarian cancer (HGSC) is the most prevalent type of the most lethal gynecologic malignancy: ovarian cancer ${ }^{1,5,6}$. The five-year survival rate for women diagnosed with high-grade serous ovarian cancer is between $30-40 \%$ and has not changed significantly in decades $^{5,6}$. Understanding how the gene expression of tumors differs from the organ from which it is derived, and how tumor transcriptomes differ when compared based on patient survival outcomes can provide insights into the mechanisms by which cancers are initiated and maintained. We used published microarray data ${ }^{2-4}$ to identify differentially expressed genes in HGSC. The Frizzled class 7 receptor emerged as among the genes most differentially expressed in HGSC tumors, both when comparing tumor to control ovarian tissue and when comparing patient tumor global gene expression profiles based on survival outcomes.

\section{Methods}

We used microarray data from datasets GSE140082², GSE124766³ and GSE146556 ${ }^{4}$ for this differential gene expression analysis of high-grade serous carcinomas. The Benjamini and Hochberg method of p-value adjustment was used for ranking of differential expression but raw p-values were used for assessment of statistical significance of global differential expression. Log-transformation of data was auto-detected, and the NCBI generated category of platform annotation was used. GSE140082² was generated using Illumina HumanHT-12 WG-DASL V4.0 R2 expression beadchip technology with $n=31$ for tumors from patients with progressionfree survival less than 200 days and $n=36$ for tumors from patients with progression-free survival greater than 1000 days. GSE124766³ was generated using Agilent-014850 Whole 
Human Genome Microarray 4x44K G4112F with $n=3$ of for normal ovarian tissue and $n=8$ for tumors from patients with high-grade serous ovarian cancer. GSE146556 ${ }^{4}$ was generated using

Affymetrix Human Gene 1.0 ST Array with $n=3$ for normal ovarian tissue and $n=40$ for tumors from patients with high-grade serous ovarian cancer. GEO2R provides mRNA expression levels only for the top 250 most differentially expressed genes. A statistical test was performed to evaluate whether Fzd7 expression was significantly different when comparing tumor tissue to normal ovary, or when comparing tumor tissue based on progression-free survival greater than 1100 days or less than 200 days using a two-tailed, unpaired t-test with Welch's correction. GEO2R provides exact mRNA expression levels only for the top 250 most differentially expressed genes. We used PRISM for all statistical analyses (Version 8.4.0)(455).

\section{Results}

We mined published microarray data in high-grade serous ovarian cancer (HGSC), the type of ovarian cancer responsible for $70-80 \%$ of deaths resulting from the most lethal gynecologic malignancy, to identify differentially expressed genes both based on survival outcomes $^{2}$ and when comparing tumor to the normal ovary ${ }^{3,4}$.

Fzd7 expression in HGSC associates with survival outcomes.

To identify genes associated with greater patient survival in ovarian cancer, we used tumor microarray data from patients enrolled in the ICON7 trial to compare global gene expression profiles of tumors from patients surviving less than 200 days to that of patients

PAGE 3 OF 14 
surviving greater than 1100 days $^{2}$. We found that the Frizzled class 7 receptor encoded by Fzd7 was among the genes most differentially expressed in HGSC based on survival outcomes (Table 1 and Figure 1). When sorting all transcripts measured by microarray based on significance in change in expression between tumors from women with PFS greater than 1100 days or PFS less than 200 days, Fzd7 ranked 163 out of 29377 total transcripts. Differential expression of Fzd7 in HGSC tumors based on patient survival less than 200 days or greater than 1100 days was statistically significant (Table $1 ; p=0.0025901)$.

Fzd7 is differentially expressed in ovarian tumors from women diagnosed with HGSC.

We used separate microarray data ${ }^{3}$ to identify genes associated with the HGSC tumor state by comparing primary HGSC tumors to the normal ovary. We identified Fzd7 as among the genes whose expression was most different when comparing primary HGSC tumors to normal ovarian tissue (Table 2). When sorting all of the transcripts measured by microarray based on change in expression between HGSC and the normal ovary, Fzd7 ranked 411 out of 41093 total transcripts (Table 2). Differential expression of Fzd7 in HGSC tumors was statistically significant (Table 2; 6.31E-05).

We analyzed a second microarray dataset ${ }^{4}$ generated using normal ovarian tissues and tumors from women diagnosed with HGSC to determine whether differential expression of Fzd7 could be observed in tumors from a different group of patients. We again found that Fzd7 was differentially expressed in primary HGSC tumors (Table 3). When sorting all of the transcripts measured by microarray based on change in expression between HGSC and the normal ovary, in 
this data set, Fzd7 ranked 3060 out of 29088 total transcripts (Table 3). Differential expression of Fzd7 in HGSC tumors was statistically significant (Table $3 ; p=3.4 \mathrm{E}-03$ ).

Fzd7 is expressed at significantly lower levels in HGSC tumors from women with progression-free survival greater than 1100 days.

We obtained exact mRNA expression levels ${ }^{2}$ for the differentially expressed Fzd7 transcript in tumors from with progression-free survival greater than 1100 days when compared to tumors from women with PFS less than 200 days. Fzd7 was expressed at significantly lower levels in high-grade serous tumors from women with PFS greater than 1100 days when compared to tumors from women with PFS less than 200 days (Figure 1; $p=0.0188)$. We calculated a mean fold change of $.9146 \pm 0.1040$ in Fzd7 expression when comparing HGSC tumors from women with PFS greater than 11100 days when compared to tumors from women with PFS less than 200 days (Table 1).

Thus, we found using published microarray data ${ }^{2}$ that Fzd7 was among the most differentially expressed genes when comparing primary HGSC tumors based on patient survival, expressed at significantly lower levels in the tumors of patients surviving greater than 200 days when compared to that of patients surviving less than 1100 days. We also observed significant differential expression of Fzd7 in HGSC tumors when compared to the normal ovary ${ }^{3,4}$.

PAGE 5 OF 14 


\section{Discussion}

The Frizzled class 7 receptor is a membrane receptor for the Wnt signaling pathway and the most abundantly expressed FZD gene in human embryonic stem cells 7,8 . Fzd7 functions in maintenance of pluripotency in human embryonic stem cells by transmitting a Wnt3a signal through $\boldsymbol{\beta}$-catenin to maintain hES cells in an undifferentiated state ${ }^{7,8}$.

In addition to Wnt3a, another described ligand of Fzd7 is Wnt7a. Wnt7a signaling can activate the planar-cell-polarity pathway during stem cell expansion in skeletal muscle repair. Addition of Wnt7a to differentiated $\mathrm{C} 2 \mathrm{C} 12$ myotubes resulted in increased myotube diameter, indicating that Wnt7a could drive hypertrophy in differentiated myofibers 9 . Wnt7a signaling leads to activation of the mTOR/protein kinase Akt pathway. In $\mathrm{C} 2 \mathrm{C} 12$ myotubes, Fzd7 can be found in complex with phosphoinositide-3-kinase (PI3K) and the G protein Gos ${ }^{9}$.

Fzd7 is implicated in a number of human cancers. In the Stem-A sub-variety of ovarian cancer, Fzd7 expression was found to be enriched in patient tumors when compared to Stem-B, epithelial-A (Epi-A), Epi-B, or mesenchymal types, and among cell lines Fzd7 expression was relatively highest in the Stem-A cell lines PA1, CH1 and OV-17R ${ }^{10}$. Depletion of Fzd7 in these cell lines resulted in decreases in cell proliferation and an increase in percentage of cells in $\mathrm{G}_{0}$ but with no change in frequency of apoptotic cells. Importantly, Fzd7-depleted cells became more epithelial in morphology and polarized; tensile contractility increased after Fzd7 depletion, evidenced by relocalization of phosphorylated myosin light chain from sites of cell-cell 
contact to colony periphery, as well as loss of stress fibers ${ }^{10}$. A number of planar-cell-polarity genes were expressed at higher levels in Stem-A tumors compared to Stem-B tumors, including CELSR3, PRICKLE4, PFN2, PCDHB17, PTK7 and SPRY1. Fzd7 depletion by small interfering RNA (siRNA) resulted in inhibition of RhoA but activation of Rac1, and there was a significant decrease in localization of CK1 $\varepsilon$ at the $\gamma$-tubulin centrosome ${ }^{10}$. Together, the data suggested that Fzd7 influenced cell cycle and proliferation in Stem-A ovarian cancers, as well as cell migration phenotypes through the planar cell polarity and non-canonical Wnt pathways ${ }^{10}$. A separate study demonstrated that depletion of Fzd7 in the OV-17R and $\mathrm{CH} 1$ ovarian cell lines using small hairpin RNA (shRNA) depletion resulted in acquisition of epithelial morphology and the down-regulation of epithelial mesenchymal transition genes including TWIST ${ }^{11}$. The ability to avoid cell death in environments where adherence signals are deficient, anoikis resistance, was significantly decreased in cells depleted of Fzd7 and spheroid formation by OV-17R and $\mathrm{CH} 1$ cells was also reduced ${ }^{11}$.

Fzd7 promotes cell proliferation in triple negative breast cancer ${ }^{12}$. Depletion of Fzd7 in TNBC cell lines MDA-MD-231 and BT-20 resulted in decreased cell proliferation and colony formation ${ }^{12}$. One study demonstrated that Wilms' tumors (WT) could be distinguished based on response to Fzd7 blockade by Fzd7 antibody ${ }^{13}$. Fzd7 + cells sorted from Fzd7-resistant WT were highly proliferative and clonogenic with significantly increased expression of stemness genes SIX2, SALL1 and OCT-413.

PAGE 7 OF 14 
Fzd7 expression is also increased in gliomas, with expression associated with more advanced tumor stage ${ }^{14}$. Fzd7 could activate the transcriptional coactivator TAZ and proliferation of gliomas supported by Fzd7 required TAZ expression ${ }^{14}$. Patients whose glioma tumors expressed highest levels of Fzd7 had significantly shorter survival14. In the U-87MG cell line ectopically expressing Fzd7 in xenografts in vivo, tumor weight and tumor volume was significantly greater. Together, the data suggest a role for Fzd7 in promotion of tumor growth and stemness.

We found significant changes in expression of the Frizzed class 7 receptor in the tumors of patients with high-grade serous ovarian cancer. Fzd7 expression was also differentially expressed when comparing tumors based on progression-free survival, with significantly lower expression of Fzd7 in tumors from patients with progression-free survival greater than 1100 days. Differential expression of Fzd7 in HGSC should be validated in larger and separate cohorts of women with HGSC. If validated, mouse models of high-grade serous ovarian cancers $^{15-17}$ can be utilized to assess the effect of conditional expression of Fzd7 on HGSC tumor growth post-development and on survival in vivo. 


\section{References}

1. Guppy, A.E., Nathan, P.D. and Rustin, G.J., 2005. Epithelial ovarian cancer: a review of current management. Clinical Oncology, 17(6), pp.399-411

2. GSE140082. Winterhoff B, Kommoss S. Mayo Clinic, Obstetrics and Gynecology. https:// www.ncbi.nlm.nih.gov/geo/query/acc.cgi?acc=GSE140082.

3. Hoffmann, K., Berger, H., Kulbe, H., Thillainadarasan, S., Mollenkopf, H.J., Zemojtel, T., Taube, E., Darb-Esfahani, S., Mangler, M., Sehouli, J. and Chekerov, R., 2020. Stable expansion of high-grade serous ovarian cancer organoids requires a low-Wnt environment. The EMBO journal, 39(6), p.e104013

4. Zhang, W., Klinkebiel, D., Barger, C.J., Pandey, S., Guda, C., Miller, A., Akers, S.N., Odunsi, K. and Karpf, A.R., 2020. Global DNA hypomethylation in epithelial ovarian cancer: passive demethylation and association with genomic instability. Cancers, 12(3), p.76

5. Bowtell, D.D., Böhm, S., Ahmed, A.A., Aspuria, P.J., Bast Jr, R.C., Beral, V., Berek, J.S., Birrer, M.J., Blagden, S., Bookman, M.A. and Brenton, J.D., 2015. Rethinking ovarian cancer II: reducing mortality from high-grade serous ovarian cancer. Nature reviews Cancer, 15(11), pp.668-679.

6. Vaughan, S., Coward, J.I., Bast, R.C., Berchuck, A., Berek, J.S., Brenton, J.D., Coukos, G., Crum, C.C., Drapkin, R., Etemadmoghadam, D. and Friedlander, M., 2011. Rethinking ovarian cancer: recommendations for improving outcomes. Nature Reviews Cancer, 11(10), pp.719-725.

7. Melchior, K., Weiß, J., Zaehres, H., Kim, Y.M., Lutzko, C., Roosta, N., Hescheler, J. and Müschen, M., 2008. The WNT receptor FZD7 contributes to self-renewal signaling of human embryonic stem cells. Biological chemistry, 389(7), pp.897-903.

8. Fernandez, A., Huggins, I.J., Perna, L., Brafman, D., Lu, D., Yao, S., Gaasterland, T., Carson, D.A. and Willert, K., 2014. The WNT receptor FZD7 is required for maintenance of the pluripotent state in human embryonic stem cells. Proceedings of the National Academy of Sciences, 111(4), pp.1409-1414.

9. Von Maltzahn, J., Bentzinger, C.F. and Rudnicki, M.A., 2012. Wnt7a-Fzd7 signalling directly activates the Akt/mTOR anabolic growth pathway in skeletal muscle. Nature cell biology, 14(2), pp.186-191.

PAGE 9 OF 14 
10.Asad, M., Wong, M.K., Tan, T.Z., Choolani, M., Low, J., Mori, S., Virshup, D., Thiery, J.P. and Huang, R.Y., 2014. FZD7 drives in vitro aggressiveness in Stem-A subtype of ovarian cancer via regulation of non-canonical Wnt/PCP pathway. Cell death \& disease, 5(7), pp.e1346-e1346.

11.Tan, M., Asad, M., Heong, V., Wong, M.K., Tan, T.Z., Ye, J., Kuay, K.T., Thiery, J.P., Scott, C. and Huang, R.Y.J., 2019. The FZD 7-TWIST 1 axis is responsible for anoikis resistance and tumorigenesis in ovarian carcinoma. Molecular oncology, 13(4), pp.757-780.

12.Yang, L., Wu, X., Wang, Y., Zhang, K., Wu, J., Yuan, Y.C., Deng, X., Chen, L., Kim, C.C.H., Lau, S. and Somlo, G., 2011. FZD7 has a critical role in cell proliferation in triple negative breast cancer. Oncogene, 30(43), pp.4437-4446.

13.Pode-Shakked, N., Harari-Steinberg, O., Haberman-Ziv, Y., Rom-Gross, E., Bahar, S., Omer, D., Metsuyanim, S., Buzhor, E., Jacob-Hirsch, J., Goldstein, R.S. and Mark-Danieli, M., 2011. Resistance or sensitivity of Wilms' tumor to anti-FZD7 antibody highlights the Wnt pathway as a possible therapeutic target. Oncogene, 30(14), pp.1664-1680.

14.Qiu, X., Jiao, J., Li, Y. and Tian, T., 2016. Overexpression of FZD7 promotes glioma cell proliferation by upregulating TAZ. Oncotarget, 7(52), p.85987.

15.Harrington, B.S., He, Y., Davies, C.M., Wallace, S.J., Adams, M.N., Beaven, E.A., Roche, D.K., Kennedy, C., Chetty, N.P., Crandon, A.J. and Flatley, C., 2016. Cell line and patientderived xenograft models reveal elevated $\mathrm{CDCP} 1$ as a target in high-grade serous ovarian cancer. British journal of cancer, 114(4), pp.417-426.

16.Perets, R., Wyant, G.A., Muto, K.W., Bijron, J.G., Poole, B.B., Chin, K.T., Chen, J.Y.H., Ohman, A.W., Stepule, C.D., Kwak, S. and Karst, A.M., 2013. Transformation of the fallopian tube secretory epithelium leads to high-grade serous ovarian cancer in Brca; $\mathrm{Tp} 53$; Pten models. Cancer cell, 24(6), pp.751-765.

17.Kim, J., Coffey, D.M., Creighton, C.J., Yu, Z., Hawkins, S.M. and Matzuk, M.M., 2012. High- grade serous ovarian cancer arises from fallopian tube in a mouse model. Proceedings of the National Academy of Sciences, 109(10), pp.3921-3926. 


\section{Table 1: Fzd7 is differentially expressed in high-grade serous ovarian carcinomas when} comparing tumors based on progression-free survival greater than 1100 days or less than 200 days.

The rank of differential expression, the probe/transcript ID, the p-value with respect to global differential expression, $t$, a moderated $t$-statistic, $B$, the log-odds of differential expression between the two groups compared, the fold change of Fzd7 in patients with PFS $>1100$ days when compared to patients with PFS $<200$ days, the gene and gene name are listed in this chart. 


\begin{tabular}{|c|c|c|c|c|c|c|}
\hline Rank & ID & $p$-value & $\mathbf{t}$ & B & Gene & Gene name \\
\hline 411 & A_23_P209449 & 6.31E-05 & -6.0464001 & 2.06592 & FZD7 & $\begin{array}{l}\text { frizzled class } \\
\text { receptor } 7\end{array}$ \\
\hline
\end{tabular}

Table 2: Fzd7 is differentially expressed in high-grade serous ovarian carcinomas.

The rank of differential expression, the probe/transcript ID, the p-value with respect to global differential expression, $t$, a moderated $t$-statistic, $B$, the log-odds of differential expression between the two groups compared, the gene and gene name are listed in this chart.

PAGE 12 OF 14 


\begin{tabular}{|c|c|c|c|c|c|c|}
\hline Rank & ID & $p$-value & $t$ & B & Gene & Gene name \\
\hline 3060 & 8047487 & $3.4 \mathrm{E}-03$ & -3.0955394 & -2.2003367 & FZD7 & $\begin{array}{l}\text { frizzled class } \\
\text { receptor } 7\end{array}$ \\
\hline
\end{tabular}

Table 3: Fzd7 is differentially expressed in high-grade serous ovarian carcinomas.

The rank of differential expression, the probe/transcript ID, the p-value with respect to global differential expression, $t$, a moderated $t$-statistic, $B$, the log-odds of differential expression between the two groups compared, the gene and gene name are listed in this chart.

PAGE 13 OF 14 


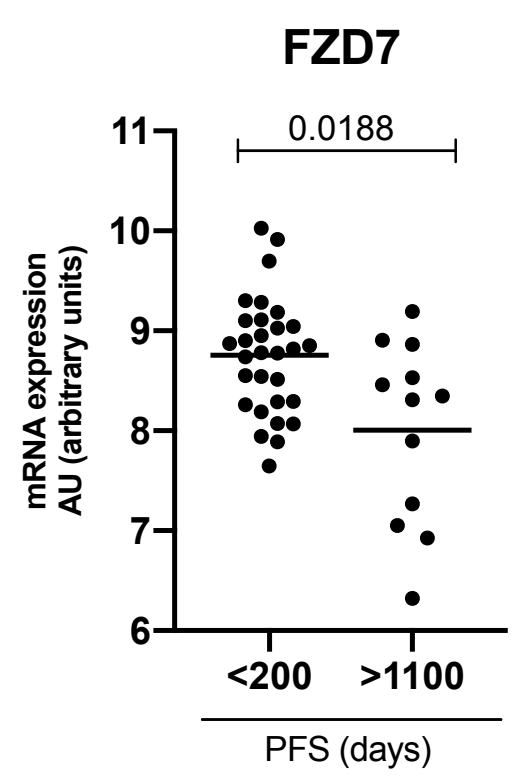

Figure 1: Fzd7 is expressed at significantly lower levels in HGSC tumors of patients surviving greater than $\mathbf{1 1 0 0}$ days when compared to that of tumors from patients surviving less than 200 days.

The mRNA expression of Fzd7 in tumors from HGSC patients surviving less than 200 days (left) and in the primary tumors of HGSC patients surviving greater than 1100 days (right) is represented with mean mRNA expression level marked and the result of a statistical test, a pvalue, listed above. 